BMJ Open Diabetes Research \& Care

\title{
FOXN3 hyperglycemic risk allele and insulin sensitivity in humans
}

\author{
Melissa L Erickson, ${ }^{1}$ Santhosh Karanth, ${ }^{2}$ Eric Ravussin, ${ }^{1}$ Amnon Schlegel ${ }^{2}$
}

To cite: Erickson ML, Karanth S, Ravussin E, et al. FOXN3 hyperglycemic risk allele and insulin sensitivity in humans. BMJ Open Diab Res Care 2019;7:e000688. doi:10.1136/ bmjdrc-2019-000688

Received 15 April 2019 Revised 6 August 2019 Accepted 9 August 2019
Check for updates

(C) Author(s) (or their employer(s)) 2019. Re-use permitted under CC BY-NC. No commercial re-use. See rights and permissions. Published by BMJ.

${ }^{1}$ Pennington Biomedical Research Center, Louisiana State University System, Baton Rouge, Louisiana, USA ${ }^{2}$ University of Utah Molecular Medicine Program and the Departments of Internal Medicine, Biochemistry, and Nutrition and Integrative Physiology, University of Utah School of Medicine, Salt Lake City, Utah, USA

Correspondence to Dr Amnon Schlegel; amnons@u2m2.utah.edu

\section{ABSTRACT}

Objective The rs8004664 variation within the FOXN3 gene is significantly and independently associated with fasting blood glucose in humans. We have previously shown that the hyperglycemia risk allele $(A)$ increases FOXN3 expression in primary human hepatocytes; overexpression of human FOXN3 in zebrafish liver increases fasting blood glucose; and heterozygous deletion of the zebrafish ortholog foxn3 decreases fasting blood glucose. Paralleling these model organism findings, we found that rs8004664 AIA homozygotes had blunted glucagon suppression during an oral glucose tolerance test. Here, we test associations between insulin sensitivity and the rs8004664 variation.

Research design and methods 92 participants $\left(49 \pm 13\right.$ years, body mass index: $32 \pm 6 \mathrm{~kg} / \mathrm{m}^{2}, 28$ with and 64 without type 2 diabetes mellitus) were genotyped at rs8004664. Insulin sensitivity was measured by the euglycemic-hyperinsulinemic clamp technique.

Results The "A" allele frequency was $59 \%$; the protective (G) allele frequency was $41 \%$ (AIA: $n=29 ; \mathrm{GIG}: n=12$; AIG: $\mathrm{n}=50$ ). Clamp-measured glucose disposal rate (GDR) was not different by genotype ( $F=0.046, p=0.96)$ or by " $A$ " allele carrier $(p=0.36)$. Female GIG homozygotes had better insulin sensitivity compared to female "A" allele carriers (GDR; GIG: $9.9 \pm 3.0$ vs AIA+AlG: $7.1 \pm 3.0 \mathrm{mg} / \mathrm{kg}$ fat-free mass+17.7/min; $p=0.04$ ). Insulin sensitivity was not different by genotype or by "A" allele carriers.

Conclusion The rs8004664 variation within the FOXN3 gene may modulate insulin sensitivity in women.

\section{INTRODUCTION}

In a large cohort of non-diabetic subjects, the single nucleotide variation rs8004664 within the first intron of the FOXN3 gene in humans was found to be significantly and independently associated with fasting blood glucose. ${ }^{1}$ The molecular mechanism for how this variation increases the fasting blood glucose set-point remains elusive; nevertheless, we previously showed that the fasting hyperglycemia allele at rs804464 increases FOXN3 expression in primary human hepatocytes. ${ }^{2}$ We modeled this increased liver FOXN3 expression by over-expressing the human FOXN3 cDNA in zebrafish livers and observed an increase in fasting blood glucose without any additional dietary challenge. Since FOXN3 is a transcriptional repressor, ${ }^{3}$ we performed whole transcriptome analyses

\section{Significance of this study}

What is already known about this subject?

- The fasting hyperglycemia risk allele of the rs8004664 variation in the FOXN3 gene increases expression of FOXN3 in the liver and is associated with blunted suppression of glucagon during an oral glucose tolerance test.

- Over-expression of FOXN3 in liver increases, whereas knockout of the FOXN3 ortholog decreases fasting blood glucose in zebrafish.

What are the new Findings?

- The role of FOXN3 in modulating insulin sensitivity was not known.

- We genotyped a cohort of subjects who underwent high-dose euglycemic-hyperinsulinemic clamp for the rs8004664.

- The fasting hyperglycemia variant of rs8004664 was associated with increased glucose disposal in female subjects, but not in men or the combined cohort.

How might these results change the focus of research or clinical practice?

- FOXN3 may modulate insulin sensitivity in a sexually dimorphic manner.

in livers over-expressing FOXN3: the $M Y C$ ortholog mycb transcript, which encodes a driver of liver glucose utilization during fasting, ${ }^{4}$ was strongly down-regulated. We showed that FOXN3 directly represses $M Y C$ expression. ${ }^{2}$ This indicates that liver FOXN3 increases fasting blood glucose by repressing a driver of liver glucose utilization, providing more glucose for export from the liver. ${ }^{2}$

In follow-up investigations, we found that glucagon injection into mice rapidly decreases liver FOXN3 protein, indicating hormonal regulation of FOXN3. When we prepared a viable loss-of-function mutation in the orthologous foxn3 gene, we observed decreased fasting blood glucose, blood glucagon, and alpha cell mass. ${ }^{5}$ Concordantly, over-expression of human FOXN3 in zebrafish liver increased alpha cell mass in the zebrafish endocrine pancreas. In this second study, we also explored the effect of the rs8004664 
variation on oral glucose tolerance in a large cohort of human subjects: rs8004644 hyperglycemia risk allele carriers show diminished suppression of glucagon over the oral glucose tolerance test (as reflected by decreased area below baseline), but show no differences in fasting glucagon. ${ }^{5}$

Our working model for how FOXN3 regulates fasting glucose does not exclude a potential role for insulin sensitivity, and therefore glucose disposal rate (GDR) during a glucose clamp. Here, we tested in a cohort of adults with and without type 2 diabetes whether the rs 8004664 variation modulates insulin-mediated glucose uptake by examining associations between rs8004664 variants and insulin sensitivity measured by the gold-standard euglycemic-hyperinsulinemic clamp technique. ${ }^{6}$

\section{METHODS}

\section{Study population}

In this single group cross-sectional design, 92 participants who previously underwent a euglycemic-hyperinsulinemic clamp ${ }^{6}$ were genotyped at the rs 8004664 variant. Participants were initially part of a larger prospective study called "The Pennington Center Longitudinal Study" designed to assess the effects of obesity and lifestyle factors on the chronic disease development, including type 2 diabetes mellitus. The current study utilized a subset of participants (with available DNA) from the original cohort, consisting of 92 white adults. Participants were categorized as having type 2 diabetes by self-report ("yes" response to having diabetes) or by fasting plasma glucose $\geq 126 \mathrm{mg} / \mathrm{dL}$, on study visit.

\section{Anthropometrics/body composition}

As previously described, ${ }^{6}$ metabolic body weight and height were measured, and body mass index was calculated from these respective values. Per cent body fat was determined from dual-energy X-ray absorptiometry (DXA, Hologics QDR 4500A; Hologics, Bedford, MA, USA). Fat mass and fat-free mass (FFM) were calculated from DXA-measured whole body per cent fat and metabolic body weight.

\section{Insulin sensitivity}

Peripheral insulin sensitivity was measured using the gold-standard euglycemic-hyperinsulinemic clamp technique, as previously described. ${ }^{6}$ Briefly, plasma glucose was clamped between 90 and $100 \mathrm{mg} / \mathrm{dL}$ during continuous insulin infusion $\left(120 \mathrm{mU} / \mathrm{m}^{2} / \mathrm{min}\right)$. GDRs presented herein are normalized for metabolic size of the participant (FFM +17.7) as well as average insulin concentrations during steady state of the clamp procedure. ${ }^{7}$ Average insulin concentrations measured during steady state of the clamp for the entire study cohort was $227.1 \pm 68 \mu \mathrm{U} / \mathrm{mL}$. Fasting glucose, insulin, lipids and triglycerides were measured from a fasting blood draw before the clamp procedure.
Table 1 Participant characteristics and glucose disposal rate by rs 8004664 genotype

\begin{tabular}{|c|c|c|c|}
\hline Characteristic & $\begin{array}{l}\text { Genotype } \\
\mathrm{A}|\mathrm{A}+\mathrm{A}| \mathrm{G} \\
(\mathrm{n}=79)\end{array}$ & $\begin{array}{l}\text { Genotype } \\
G \mid G \\
(n=13)\end{array}$ & $\mathrm{P}$ value \\
\hline Age (y) & $50 \pm 13$ & $46 \pm 15$ & 0.32 \\
\hline $\operatorname{Sex}(M / F)$ & $36 / 43$ & $7 / 6$ & NA \\
\hline BMI $\left(\mathrm{kg} / \mathrm{m}^{2}\right)$ & $33 \pm 6$ & $32 \pm 7$ & 0.91 \\
\hline Body weight (kg) & $93 \pm 19$ & $93 \pm 22$ & 0.99 \\
\hline FFM (kg) & $58.9 \pm 11.7$ & $59.6 \pm 14.0$ & 0.85 \\
\hline Fasting glucose (mg/dL) & $102 \pm 17$ & $102 \pm 12$ & 0.97 \\
\hline Fasting insulin ( $\mu \mathrm{U} / \mathrm{mL})$ & $15 \pm 7$ & $17 \pm 21$ & 0.56 \\
\hline HOMA-IR & $3.9 \pm 2.1$ & $4.6 \pm 6.2$ & 0.40 \\
\hline DXA (\% Fat) & $36 \pm 9$ & $35 \pm 8$ & 0.83 \\
\hline Cholesterol (mg/dL) & $203 \pm 42$ & $200 \pm 44$ & 0.79 \\
\hline HDL (mg/dL) & $50 \pm 12$ & $53 \pm 17$ & 0.49 \\
\hline LDL (mg/dL) & $120 \pm 36$ & $117 \pm 43$ & 0.80 \\
\hline Triglycerides (mg/dL) & $165 \pm 115$ & $148 \pm 118$ & 0.61 \\
\hline Diabetes status (Y/N) & $25 / 54$ & $3 / 10$ & NA \\
\hline $\begin{array}{l}\text { Glucose disposal (mg/kg } \\
\text { FFM +17.7/min) }\end{array}$ & $6.7 \pm 2.9$ & $7.5 \pm 3.1$ & 0.36 \\
\hline
\end{tabular}

BMI, body mass index; FFM, fat-free mass; HDL, high-density lipoprotein; LDL, low-density lipoprotein.

\section{Genotyping}

DNA was extracted from buffy coat of blood samples (Qiagen Puregene Blood Core Kit \#158389). Genotyping of the rs8004664 variant was completed using a TaqMan SNP Genotyping Assay (ThermoFisher ID C__29386020_10) as previously described. ${ }^{5}$

\section{Statistical analysis}

Data are presented as mean $\pm \mathrm{SD}$, unless otherwise noted. SPSS (IBM V. 25) was used for statistical analysis. Participants were grouped by genotype $(\mathrm{GlG}, \mathrm{AlG}$, and $\mathrm{AlA})$, and differences in metabolic outcomes were tested using one-way analysis of variance. Secondarily, participants were grouped by hyperglycemic risk-carrying allele versus protective allele homozygotes (AlG+AlG combined vs $G \mid G)$, and differences were tested using unpaired Student's t-test. Significance was accepted at $p=0.05$. To explore the role of type 2 diabetes status on the relationship between genetic variants and metabolic outcomes, participants were categorized by diabetes status and a similar analysis was performed among these two groups (those with and without type 2 diabetes). Furthermore, the relationship between genetic variants and metabolic outcomes was also performed separately among women and men.

\section{RESULTS}

Participant characteristics are shown in table 1 . No baseline differences were observed. Of the 92 participants, 29 were homozygous for the fasting hyperglycemia risk 
allele (AIA), 13 were homozygous for the protective allele $(\mathrm{G} \mid \mathrm{G})$, and 50 participants were heterozygous $(\mathrm{AlG})$. The high-risk allele (A) frequency was $59 \%$ and the protective allele frequency $(G)$ was $41 \%$. No differences were observed in GDR when grouped by genotype $(\mathrm{F}=0.046$, $\mathrm{p}=0.96$ ) or when grouped by carrier of hyperglycemic risk allele versus homozygotes for the protective allele "G" ( $\mathrm{p}=0.36$; table 1$)$.

Among those with type 2 diabetes $(n=28)$, no significant differences were observed in GDR by genotype group $(\mathrm{F}=0.852, \mathrm{p}=0.44)$ or by carrier of hyperglycemic risk allele $(\mathrm{p}=0.54)$. Similarly, among those without type 2 diabetes $(\mathrm{n}=64)$, no significant differences were observed in GDR grouped by genotype $(\mathrm{F}=0.149, \mathrm{p}=0.86)$ or by carrier of hyperglycemic risk allele $(\mathrm{p}=0.61)$. Insulin concentrations were not different by genotype group (INS, G|G: $261 \pm 149.7$ vs AlA+AlG: 221.5 $\pm 41.6 \mu \mathrm{U} / \mathrm{mL}$; $\mathrm{p}=0.05)$ although trending. This is driven by a single outlier within the GG group. Re-analysis after outlier removal shows similar means between groups (INS, G|G: $223.8 \pm 69.9$ vs $\mathrm{AlA}+\mathrm{AlG}: 221.5 \pm 41.6 \mu \mathrm{U} / \mathrm{mL} ; \mathrm{p}=0.87)$. Insulin concentrations were not different between males and females (INS, males: $235.6 \pm 83.5$ vs female: $220.3 \pm 42.8$ $\mu \mathrm{U} / \mathrm{mL} ; \mathrm{p}=0.26)$.

Female homozygotes for the protective allele "G" had significantly higher GDR (indicative of increased insulin sensitivity) compared to female carriers of the hyperglycemic-risk allele "A" (GIG: 9.9 \pm 3.0 vs AlA+AG: $7.1 \pm 3.0 \mathrm{mg} /$ $\mathrm{kgFFM}+17.7 / \mathrm{min} ; \mathrm{p}=0.04)$. Homeostatic model assessment of insulin resistance (HOMA-IR) was however not different between groups $(\mathrm{p}=0.33)$. However, re-analysis of data after removal of an outlier with excessive hyperinsulinemia revealed a significant difference (GIG: $1.6 \pm 0.8 \mathrm{vs}$ $\mathrm{AlA}+\mathrm{AG}$ : 3.8 $\pm 1.9 ; \mathrm{p}=0.02)$. When grouped by genotype, no differences were observed in GDR $(\mathrm{F}=2.310, \mathrm{p}=0.11)$ or HOMA-IR ( $\mathrm{F}=0.507, \mathrm{p}=0.61$ ). Among men, no significant differences were observed in GDR grouped by genotype $(\mathrm{F}=0.592, \mathrm{p}=0.56)$ or by high-risk carrier $(\mathrm{p}=0.43)$.

\section{DISCUSSION}

This is the first study to examine the links between FOXN3 genetic variants and insulin sensitivity measured by the euglycemic-hyperinsulinemic clamp technique in humans. GDR (insulin sensitivity) was not statistically different by genotype $(\mathrm{G} \mid \mathrm{G}, \mathrm{AlG}$, and $\mathrm{A} \mid \mathrm{A})$, or when grouped by hyperglycemic risk-carrying allele (GIG vs $\mathrm{A}|\mathrm{G}+\mathrm{A}| \mathrm{A})$. Among women, protective allele homozygotes $(G \mid G)$ had significantly higher GDR (indicative of better insulin sensitivity) compared to carriers of the hyperglycemic high-risk allele, suggesting a potential role for sex in modulating the relationship between genotype and glucose metabolism. Indeed, we observed a greater increase in fasting blood glucose when FOXN3 was over-expressed in female zebrafish liver. ${ }^{2}$ The findings herein are supportive but should be interpreted cautiously due the limited sample size (G|G: $n=6$; $A|G+A| A: n=43)$.
While the rs8004664 fasting hyperglycemia variation within the FOXN3 gene does not appear to impact glucose disposal, the encoded protein's role in the development of type 2 diabetes merits further investigation. In particular, FOXN3's role in responding to and modulating glucagon function will be the subject of future studies. ${ }^{5}$ It is clear from a large body of preclinical and early clinical work that total genetic or pharmacological blockade of hepatic glucagon action is not a viable therapeutic approach. While blood glucose is lowered, hepatic transaminases, circulating triglycerides, and low-density lipoprotein cholesterol are elevated; and alpha cell hyperplasia occurs when glucagon receptor-blocking antibodies are administered. ${ }^{8}$ Thus, a more thorough understanding of the effects and effectors of glucagon action could lead to the development of more selective, glucagon-leveraging therapies for type 2 diabetes mellitus. ${ }^{9}{ }^{10}$

In summary, we provide novel data on the relationship between the FOXN3 rs8004664 variant and insulin sensitivity measured by the euglycemic-hyperinsulinemic clamp technique in humans. We did not find differences among genotype, suggesting that other physiological factors may be responsible for modulating fasting blood glucose. To further define the liver FOXN3-glucagon axis, future studies should explore links between this variant and other physiological modulators of glycemia including hepatic insulin sensitivity, glucagon secretion, and insulin secretion. Critically, FOXN3 is expressed in several tissues, often by more than one cell type in a tissue. ${ }^{11}$ Thus, a combination of human physiological characterization of the effect of the rs8004664 variation and a systematic effort to modulate FOXN3 expression in different cell types in model organisms is warranted.

Contributors MLE analyzed and interpreted data, and drafted manuscript. SK conceptualized study, collected and interpreted data, and edited the manuscript. ER conceptualized study, collected and interpreted data, and edited the manuscript. AS conceptualized study, collected and interpreted data, and edited the manuscript. ER is the guarantor of the study, having full access to all study data; he had the final responsibility for data integrity, accuracy of data analysis, and decision to submit for publication. All authors have approved the final version of this manuscript.

Funding This study was funded in part from the National Institutes of Health (grants P30DK072476; ER), and funds from the University of Utah Molecular Medicine Program and University of Utah School of Medicine, Department of Internal Medicine (AS).

Competing interests None declared.

Patient consent for publication Not required.

Ethics approval The study was performed in accordance with the principles contained within the Declaration of Helsinki. The protocol was approved by the Institutional Review Board of the Pennington Biomedical Research Center. All participants provided informed, written consent prior to study participation.

Provenance and peer review Not commissioned; externally peer reviewed.

Data availability statement Data are available upon reasonable request.

Open access This is an open access article distributed in accordance with the Creative Commons Attribution Non Commercial (CC BY-NC 4.0) license, which permits others to distribute, remix, adapt, build upon this work non-commercially, and license their derivative works on different terms, provided the original work is properly cited, appropriate credit is given, any changes made indicated, and the use is non-commercial. See: http://creativecommons.org/licenses/by-nc/4.0/. 


\section{REFERENCES}

1. Manning AK, Hivert M-F, Scott RA, et al. A genome-wide approach accounting for body mass index identifies genetic variants influencing fasting glycemic traits and insulin resistance. Nat Genet 2012;44:659-69.

2. Karanth S, Zinkhan EK, Hill JT, et al. FOXN3 regulates hepatic glucose utilization. Cell Rep 2016;15:2745-55.

3. Scott KL, Plon SE. CHES1/FOXN3 interacts with Skiinteracting protein and acts as a transcriptional repressor. Gene 2005;359:119-26.

4. Valera A, Pujol A, Gregori X, et al. Evidence from transgenic mice that Myc regulates hepatic glycolysis. Faseb J 1995;9:1067-78.

5. Karanth S, Adams JD, Serrano MdeLA, et al. A hepatocyte FOXN3- $\alpha$ cell glucagon axis regulates fasting glucose. Cell Rep 2018;24:312-9.
6. Tam CS, Xie W, Johnson WD, et al. Defining insulin resistance from Hyperinsulinemic-Euglycemic clamps. Diabetes Care 2012;35:1605-10.

7. Lillioja S, Bogardus C. Obesity and insulin resistance: lessons learned from the Pima Indians. Diabetes Metab Rev 1988;4:517-40.

8. Gromada J, Chabosseau P, Rutter GA. The $\alpha$-cell in diabetes mellitus. Nat Rev Endocrinol 2018;14:694-704.

9. Wewer Albrechtsen NJ, Pedersen J, Galsgaard KD, et al. The liveralpha cell axis and type 2 diabetes. Endocr Rev 2019. doi:10.1210/ er.2018-00251. [Epub ahead of print: 28 Mar 2019].

10. Finan B, Capozzi ME, Campbell JE. Repositioning glucagon action in the physiology and pharmacology of diabetes. Diabetes 2019:dbi190004.

11. Uhlén M, Fagerberg L, Hallström BM, et al. Proteomics. tissue-based map of the human proteome. Science 2015;347:1260419. 